\title{
Interval Lymph Node
}

National Cancer Institute

\section{Source}

National Cancer Institute. Interval Lymph Node. NCI Thesaurus. Code C12984.

One of the nodes that lie along the course of a lymphatic vessel between a primary melanoma site and a recognized node field. (from Medscape) 